Second Generation Wavelets and Applications 
Maarten Jansen and Patrick Oonincx

\section{Second Generation \\ Wavelets and \\ Applications}

With 58 Figures

算Springer 
Maarten Jansen, MSc Engineering, PhD Computer Science

Department of Mathematics and Computer Science

TU Eindhoven, PO Box 513

5600 MB Eindhoven, The Netherlands

and

Department of Computer Science

K.U. Leuven, Celestijnenlaan 200A

3001 Leuven, Belgium

Patrick Oonincx, MSc Mathematics, PhD Mathematics

Royal Netherlands Naval College

PO Box 10000

1780 CA Den Helder, The Netherlands

British Library Cataloguing in Publication Data

Jansen, Maarten

Second generation wavelets and applications

1. Signal processing - Mathematics 2. Image processing -

Mathematics 3. Wavelets (Mathematics)

I. Title II. Oonincx, Patrick

$515.2^{\prime} 433$

ISBN 1852339160

Library of Congress Cataloging-in-Publication Data

Jansen, Maarten.

Second generation wavelets and applications / Maarten Jansen and Patrick Oonincx.

p. cm.

Includes bibliographical references and index.

ISBN 1-85233-916-0 (alk. paper)

1. Signal processing-Mathematics. 2. Image processing-Mathematics. 3. Wavelets

(Mathematics) I. Oonincx, Patrick. II. Title.

TK5102.9.J3623 2005

$515^{\prime} .2433-\mathrm{dc} 22$

2004058967

Apart from any fair dealing for the purposes of research or private study, or criticism or review, as permitted under the Copyright, Designs and Patents Act 1988, this publication may only be reproduced, stored or transmitted, in any form or by any means, with the prior permission in writing of the publishers, or in the case of reprographic reproduction in accordance with the terms of licences issued by the Copyright Licensing Agency. Enquiries concerning reproduction outside those terms should be sent to the publishers.

ISBN 1-85233-916-0

Springer Science+Business Media

springeronline.com

(c) Springer-Verlag London Limited 2005

The use of registered names, trademarks, etc. in this publication does not imply, even in the absence of a specific statement, that such names are exempt from the relevant laws and regulations and therefore free for general use.

The publisher makes no representation, express or implied, with regard to the accuracy of the information contained in this book and cannot accept any legal responsibility or liability for any errors or omissions that may be made.

Typesetting: Electronic text files prepared by authors

Printed in the United States of America

69/3830-543210 Printed on acid-free paper SPIN 10974018 


\section{Preface}

The ongoing success of wavelet theory and similar multiscale data decompositions is largely due to its flexibility in continuously expanding its horizon towards new applications. The first success of wavelets came in the 1980s with the continuous wavelet transform as a method in the time-scale analysis of signals. While this research went on (and still goes on), it was soon realized that the concept of time-scale analysis is closely related to the sort of signal processing that happened in so-called Laplacian pyramids. The formal definition of multiresolution, the corresponding fast wavelet transform algorithm (Mallat) and the discovery of compactly supported orthogonal wavelets (Daubechies) were major breakthroughs in the development of the discrete wavelet transform as a tool in signal and image processing. In spite of this immediate success, the classical discrete wavelet transform is somehow limited. A typical example of such a limitation is the assumption that the input has to be a regularly observed (sampled) signal, where the number of observations is a power of two. Obviously, these limitations can be dealt with by some pre- and/or post-processing of the data: think about interpolation for irregularity and symmetric extension if the number of data is not a power of two. Nevertheless, it seems more interesting if we can incorporate the grid structure and the interval boundaries into the actual construction of the multiresolution analysis. This is exactly what was aimed by 'second-generation wavelets' when they were first proposed (Sweldens), about 10 years ago.

The construction of second-generation wavelets is based on the lifting scheme. In the first instance, this scheme is an alternative implementation of the fast discrete wavelet transform. It is a bit faster than the classical repeated filterbank implementation and it allows in-place computations, i.e., no additional working memory is needed, as all steps in the algorithm may overwrite input data without making the transform irreversible. It was soon realized that this lifting scheme has another, important advantage: unlike the classical filterbank implementation, the concept is readily extensible to differently structured data. This includes not only the abovementioned irregularly observed data and data on an interval, but nearly any type of structure. One could now think, for instance, about a multiresolution analysis of DNA-molecule structures, large networks, surfaces in computer graphics applications and so on. Even for common images, it pays off using the lifting scheme, as was illustrated by the new compression standard in JPEG-2000, which makes use of lifting. Lifting also allows one to add a multiscale element to previously developed 
methods. In statistics, for instance, lifting can be used in combination with existing smoothing methods, such as spline smoothing.

Another extension, equally easy to construct with the lifting scheme, are nonlinear and data-adaptive multiscale analyses. A well known example of a nonlinear multiresolution decomposition is the integer wavelet transform that maps an array of integers to another array of integers. Data-adaptive multiresolution transforms are extensively discussed further in this book. In two dimensions, a specific data-adaptive, anisotropic approach, discussed in the last chapter, shows interesting behaviour in the neighbourhood of edges in images. While the concept of this edgeadaptive multiscale decomposition is quite easy to understand, the resulting convergence rate compares to that of more complicated approaches, such as curvelets, contourlets, edgelets and so on.

Wavelet theory and applications are situated on the edges between many fields: signal and image processing, numerical analysis and scientific computing, statistics, approximation theory and other mathematical fields. Each of these domains has its own terminology and requires a specific writing style. While people in signal processing are used to illustrations and diagrams, mathematicians prefer clear formulas to think and reason about. Samples in signal processing correspond to observations in statistics, while a sample in statistical literature is mostly the entire set of observations. Signals in signal processing are functions in mathematics. We have tried to offer both figures and formulas, fully aware that some readers might spend some time on puzzling out the meaning of each symbol in an expression or a diagram. Yet, we believe that doing so is a key to opening the doors of many papers that otherwise remain unread. Also, we tried to provide sufficient variation, in the sense that a mathematical approach is illustrated with figures and plots in a subsequent section and vice versa.

As for the mathematical depth of our approach, our point of view is to provide a guide for the exploration of the literature, rather than a fully elaborated mathematical treatment of all aspects of second-generation wavelets. We therefore omit some of the full, rigourous proofs if they would take too much space and attention. We hope to compose a clear general overview on the topic of second-generation wavelets.

Writing and even thinking this book would be a much harder job without the presence of our families.

I, Maarten Jansen, would like to express my gratitute to my wife, Gerda Claeskens, of course for her support and patience, but also for her keen curiosity and interest in my work. Her remarks after careful proofreading my chapters made a substantial improvement in readability. As she is writing a book herself now, I am sure that, if she shows the same enthusiasm there as well, that book is going to be another piece of her work she (and I!) can be proud of.

I, Patrick Oonincx, would like to thank my loving fiancee Birgit Bobeck for running our family and having patience when I was spending weekends and holidays struggling with figures and formulas. Special thanks should go to her, because for the second time in our lives she is carrying a baby while I'm writing a book and, as in the previous case, both will see daylight around the same date. Last but not least 
I have to make my apologies to my daughter Sacha for being a less playful dad in the last year. After this last sentence many formulas will be replaced by playtime. 


\section{Contents}

1. The Classical Wavelet Transform for Continuous-time and Discrete-

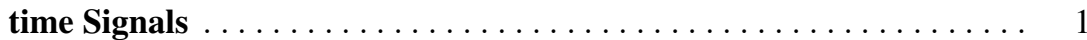

1.1 The Continuous Wavelet Transform $\ldots \ldots \ldots \ldots \ldots \ldots \ldots \ldots \ldots$

1.1.1 The Haar wavelet .......................... 3

1.1.2 The Mexican Hat Wavelet .......................... 5

1.2 The Discrete Wavelet Transform ..................... 7

1.2.1 Discretizing the Continuous Wavelet Transform.......... 7

1.2.2 Multiresolution Analysis ....................... 8

1.2.3 The Two-scale Equation $\ldots \ldots \ldots \ldots \ldots \ldots \ldots \ldots \ldots$

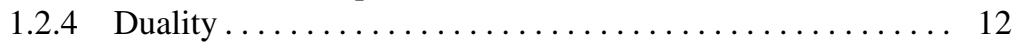

1.2.5 Vanishing Moments ...................... 13

1.3 Multiresolution Analysis and Filterbanks ................. 14

1.3.1 The Decomposition Algorithm ................... 14

1.3.2 The Reconstruction Algorithm ................ 16

1.3.3 Implementation of the Discrete Wavelet Transform for Discretetime Signals............................. 17

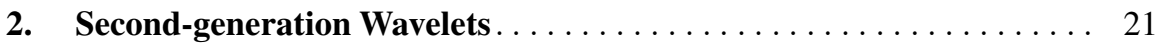

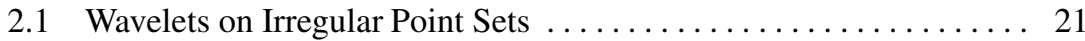

2.2 The Lifting Scheme............................. 24

2.2.1 The Haar Decomposition by Lifting .............. 24

2.2.2 The Lifting Scheme: Split, Predict and Update ......... 26

2.2 .3 Basis Functions .................................. 29

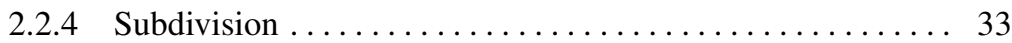

2.2.5 Lifting Existing Wavelet Transforms ............. 35



2.3 The Construction of Second-generation Wavelets ............. 40

2.3.1 Multiscale Grids ......................... 41

2.3.2 The Unbalanced Haar Transform . . . . . . . . . . . . . . . 41

2.3.3 Prediction Methods ......................... 45

2.3.4 Updates for Vanishing Moments .................. 52

2.4 Lifting in Two (and More) Dimensions ................. 53

2.4.1 Definitions and Construction of Triangulations ......... 54

2.4.2 Delaunay Triangulation ...................... 54 
2.4.3 Multiscale Triangulations ................. 55

2.4.4 Multiscale Delaunay Triangulations.............. 57



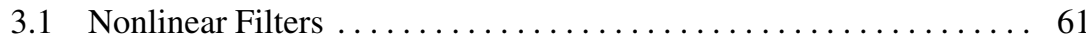

3.1.1 The Max-lifting Scheme $\ldots \ldots \ldots \ldots \ldots \ldots \ldots \ldots \ldots 6$

3.1 .2 The Median-lifting Scheme ................. 64

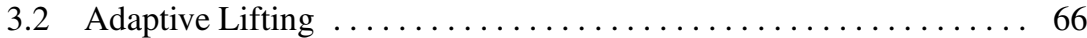

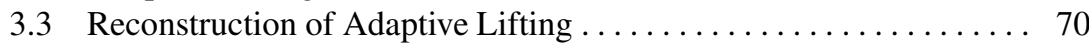

3.3.1 Automatic Perfect Reconstruction ............... 70

4. Numerical Condition $\ldots \ldots \ldots \ldots \ldots \ldots \ldots \ldots \ldots \ldots$

4.1 Stability in Wavelet Smoothing on Irregular Point Sets . . . . . . . 78

4.2 Condition from Finite to Infinite Dimensions $\ldots \ldots \ldots \ldots \ldots \ldots$

4.2 .1 Condition Numbers $\ldots \ldots \ldots \ldots \ldots \ldots \ldots \ldots \ldots \ldots \ldots \ldots$

4.2 .2 Stable Bases ...................... 82

4.3 Numerical Condition of Wavelet Transforms ............ 86

4.3.1 General Stability Criteria .................. 86

4.3.2 Smoothness, Convergence (of Approximation) and Stability 89

4.4 Numerical Condition of Lifted Wavelet Transforms . . . . . . . . . . . 91

4.4.1 Lifting of Existing Stable Schemes ............... 91

4.4.2 Numerical Condition and Primal Vanishing Moments ... . . . 92

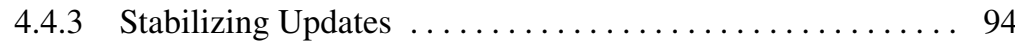



4.4.5 Splitting Strategies $\ldots \ldots \ldots \ldots \ldots \ldots \ldots \ldots \ldots \ldots \ldots$

5. Applications of Nonlinear Lifting in Imaging . . . . . . . . . . . . 103

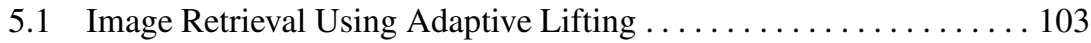

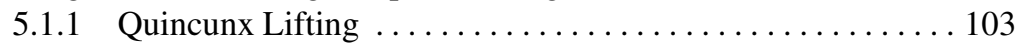

5.1 .2 Adaptive Lifting . . . . . . . . . . . . . . . . . . . 106

5.1.3 Redundant Lifting . . . . . . . . . . . . . . . . . . . . . . . . . 108

5.1 .4 Feature Vectors ........................ 109

5.1 .5 Illustration of Image Retrieval $\ldots \ldots \ldots \ldots \ldots \ldots \ldots \ldots 11$

5.2 Adaptive Splitting using Normal Offsets $\ldots \ldots \ldots \ldots \ldots \ldots \ldots \ldots$

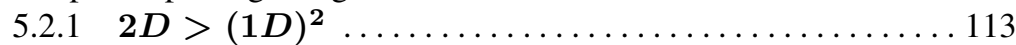

5.2 .2 Some Theoretical Background ................ 118

5.2 .3 Normal Offsets . . . . . . . . . . . . . . . . 120

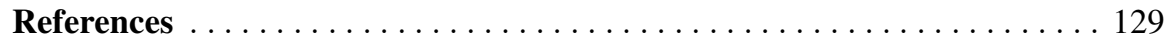

Index . . . . . . . . . . . . . . . . . . . . . . . . . 135 